\title{
Cement Thickness of Inlay Restorations Made of Lithium Disilicate, Polymer-Infiltrated Ceramic and Nano-Ceramic CAD/CAM Materials Evaluated Using 3D X-Ray Micro-Computed Tomography
}

\author{
Uzgur, Recep ; Ercan, Ertuğrul ; Uzgur, Zeynep ; Çolak, Hakan ; Yalçın, Muhammet ; Özcan, Mutlu
}

\begin{abstract}
PURPOSE To evaluate the marginal and internal cement thicknesses of inlay restorations made of various CAD/CAM materials using 3D X-ray micro-computed tomography (micro-CT) technique. MATERIALS AND METHODS Caries-free extracted mandibular molars $(\mathrm{N}=30)$ with similar size were randomly assigned to three groups ( $\mathrm{N}=10$ per group). Mesio-occlusal-distal (MOD) cavities were prepared, and inlay restorations were obtained by milling out CAD/CAM materials namely, (a) IPS: monolithic lithium disilicate (control), (b) VE: polymer-infiltrated ceramic, and (c) CS: nano-ceramic using a CAM unit. Marginal and internal cement thicknesses were measured using 3D micro-CT. Data were analyzed using 1-way ANOVA and Tukey's tests (alpha =0.05). RESULTS The mean marginal and internal cement thickness were not significant in all inlay materials ( $\mathrm{p}>0.05)$. Mean marginal cement thickness $(\mathrm{m})$ was the lowest for the IPS group $(67.54 \pm 10.16)$ followed by VE $(84.09 \pm 3.94)$ and CS $(95.18 \pm 10.58)(\mathrm{p}>0.05)$. The internal cement thickness $(\mathrm{m})$ was the lowest in the CS group (54.85 $\pm 6.94)$ followed by IPS $(60.58 \pm 9.22)$ and VE $(77.53 \pm 12.13)(\mathrm{p}>0.05)$. CONCLUSION Marginal and internal cement thicknesses of MOD inlays made of monolithic lithium disilicate, polymer-infiltrated ceramic, and nano-ceramic CAD/CAM materials were similar and all less than $100 \mathrm{~m}$, which could be considered clinically acceptable. CLINICAL SIGNIFICANCE MOD inlays made of different CAD/CAM materials presented similar cement thickness, less than $100 \mathrm{~m}$.
\end{abstract}

DOI: https://doi.org/10.1111/jopr.12521

Posted at the Zurich Open Repository and Archive, University of Zurich

ZORA URL: https://doi.org/10.5167/uzh-162675

Journal Article

Accepted Version

Originally published at:

Uzgur, Recep; Ercan, Ertuğrul; Uzgur, Zeynep; Çolak, Hakan; Yalçın, Muhammet; Özcan, Mutlu (2018). Cement Thickness of Inlay Restorations Made of Lithium Disilicate, Polymer-Infiltrated Ceramic and Nano-Ceramic CAD/CAM Materials Evaluated Using 3D X-Ray Micro-Computed Tomography. Journal of Prosthodontics, 27(5):456-460.

DOI: https://doi.org/10.1111/jopr.12521 


\section{Category of manuscript: Short Communication}

Cement Thickness of Inlay Restorations Made of Lithium disilicate, Polymer-infiltrated Ceramic and Nano-ceramic CAD/CAM Materials Evaluated Using 3D X-ray Micro-computed Tomography

Recep Uzgur, DDS, PhD¹, Ertugrul Ercan, DDS, PhD², Zeynep Uzgur, DDS, PhD¹, Hakan Çolak, DDS, $\mathrm{PhD}^{3}$, Muhammet Yalçın, DDS, $\mathrm{PhD}^{4}$, Mutlu Özcan, DDS, DMD, $\mathrm{PhD}^{5}$

${ }^{1}$ Zirve University, Faculty of Dentistry, Department of Prosthodontics, Gaziantep, Turkey

${ }^{2}$ Kırıkkale University, Faculty of Dentistry, Department of Restorative Dentistry, Kırıkkale, Turkey

${ }^{3}$ Zirve University, Faculty of Dentistry, Department of Restorative Dentistry, Gaziantep, Turkey

${ }^{4}$ İnönü University, Faculty of Dentistry, Department of Restorative Dentistry, Malatya, Turkey

${ }^{5}$ University of Zurich, Dental Materials Unit, Center for Dental and Oral Medicine, Clinic for Fixed and Removable Prosthodontics and Dental Materials Science, Zurich, Switzerland

Short title: Micro-CT evaluation of cement thickness in inlay restorations

\footnotetext{
*Correspondance:

Dr. Recep Uzgur

Zirve University

Faculty of Dentistry

Department of Prosthodontics

Gaziantep, Turkey

E-mail: ruzgur@gmail.com
} 


\section{Abstract}

Purpose: The aim of this study was to evaluate the marginal and internal cement thicknesses of inlay restorations made of various CAD/CAM materials using 3D X-ray micro-computed tomography (micro-CT) technique.

Material and Methods: Caries-free extracted mandibular molars $(\mathrm{N}=30)$ with similar size were randomly assigned to three groups ( $\mathrm{N}=10$ per group). Mesio-occlusal-distal (MOD) cavities were prepared and inlay restorations were obtained by milling out CAD/CAM materials namely, a) IPS: monolithic lithium disilicate (IPS emax CAD; control), b) VE: Polymer-infiltrated ceramic (Vita Enamic) and c) CS: Nano-ceramic (Cerasmart) using a CAM unit. Marginal and internal cement thicknesses were measured using 3D microCT (SkyScan 1172, Bruker-micro-CT). Data were analyzed using 1-way ANOVA and Tukey's tests (alpha=0.05).

Results: The mean marginal and internal cement thickness were not significant in all inlay materials $(p>$ 0.05). Mean marginal cement thickness $(\mu \mathrm{m})$ was the lowest for the IPS group $(67.54 \pm 10.16)$ followed by VE $(84.09 \pm 3.94)$ and CS $(95.18 \pm 10.58)(p>0.05)$. The internal cement thickness $(\mu m)$ was the lowest in CS group (54.85 \pm 6.94$)$ followed by IPS $(60.58 \pm 9.22)$ and VE $(77.53 \pm 12.13)(p>0.05)$.

Conclusion: Marginal and internal cement thicknesses of MOD inlays made of monolithic lithium disilicate, polymer-infiltrated ceramic and nano-ceramic CAD/CAM materials were similar being less than $100 \mu \mathrm{m}$, which could be considered clinically acceptable.

Clinical Significance: MOD inlays made of different CAD/CAM materials presented similar cement thickness being less than $100 \mu \mathrm{m}$.

Keywords CAD/CAM; Cement thickness; Micro-CT 


\section{Introduction}

Despite the advances made in modern dentistry, dental caries remains a significant problem and currently represents one of the main causes of tooth loss. In contemporary practices, moderate Class I and II carious lesions could be restored using inlay or onlay restorations ${ }^{1-4}$ that could be fabricated from a range of materials including alloy, ceramic, or resin composite. ${ }^{5}$ Long-term clinical studies still consider cast gold partial coverage as the model of excellence for restoring teeth with caries in posterior teeth. However, recently, novel materials and techniques have been introduced as non-metallic tooth-colored materials for posterior restorations. One such material is monolithic lithium disilicate all-ceramic produced using CADCAM technologies that also present high fracture resistance..$^{6,7}$

Several factors could directly influence the longevity of indirect ceramic restorations among which the quality of marginal seal and the thickness of the luting agent seems to be most relevant. ${ }^{\bar{x}}$ Marginal seal has the most substantial impact on the longevity as it ensures resistance to secondary carries and microleakage. On the other hand, the gap between the restoration and the tooth surface, filled with cement in the three-dimensional plane is also of significance for the durability of the restorations. In order to function effectively, the restoration needs mechanical support provided by the tooth substance, which becomes more crucial in the posterior teeth. ${ }^{8-10}$ Overall, three-dimensional fit is directly affected by the thickness of the cement layer. In this regard, three factors need to be taken into consideration when placing restorations, namely, the thickness, chemical structure and elastic modulus of the restoration and the cement. ${ }^{11-13}$

Marginal and internal cement thickness of a restoration could be assessed using different methods based on sectioning, replica or X-ray micro-computed tomography (micro-CT) techniques, where the latter two are considered less invasive. ${ }^{14}$ Among various methods that are employed to evaluate the fit of dental reconstructions, micro-CT is becoming increasingly common. ${ }^{15-22}$

Recently introduced CAD/CAM materials combine the durability and long-lasting colour of ceramics with the benefits of resin composite materials. Current studies reported optimal marginal and internal cement 
thickness for such materials ranging between 20 and $200 \mu \mathrm{m}^{8,23-28}$ The manufacturers however claim cement thickness within clinically acceptable levels..$^{29,30}$ In fact, cement thickness below $200 \mu \mathrm{m}$ could more effectively withstand wear of the cement at the restoration margins. ${ }^{31,32}$

The wide range of cement thickness with the recent CAD/CAM materials also relates to the measurement method. To date, micro-CT technique was not employed to assess the internal and marginal cement thickness of partial coverage restorations made of recent CAD/CAM materials. Therefore, the objective of this study was to evaluate the marginal and internal cement thickness of inlay restorations made of monolithic lithium disilicate, polymer-infiltrated ceramic and nano-ceramic CAD/CAM materials. The null hypothesis tested was that there would be no significant difference in marginal and internal cement thickness of inlay restorations fabricated from different CAD/CAM materials.

\section{Materials and Methods}

Preparation of specimens

The Ethic Committee of Kirikkale University, Faculty of Medicine, approved this research project. Cariesfree extracted mandibular molars $(\mathrm{N}=30)$ with similar size were selected. The teeth were initially examined under $\times 10$ magnification using loops and only those deemed to be free from any hypoplastic defects and cracks were selected. The teeth were thoroughly cleaned from soft tissues and stored in $0.1 \%$ thymol solution at room temperature until experiments. ${ }^{33}$ The apical parts of all teeth were embedded in an auto-polymerizing resin until the cement-enamel junction (CEJ) (Paladent RR, Heraeus Kulzer GmbH, Hanau, Germany).

The teeth were divided into three groups ( $n=10$ per group) and received an inlay manufactured from one of the following CAD/CAM materials, namely a) IPS: monolithic lithium disilicate (IPS e-max CAD, Ivoclar Vivadent, Schaan, Liechtenstein; Control group), b) VE: Polymer-infiltrated ceramic (Vita Enamic, VITA Zahnfabrik, GmbH \& Co, Bad Säckingen, Germany) and c) CS: Nano-ceramic (Cerasmart, GC Dental 
Products, Tokyo, Japan) (Table 1). A mesio-occlusal-distal (MOD) inlay cavity was prepared in each tooth with $3 \mathrm{~mm}$ isthmus width and $2.5 \mathrm{~mm}$ cavity depth. Cavities were prepared $1 \mathrm{~mm}$ above the CEJ at an overall preparation angle of 10 degrees towards the occlusal aspect and rounded shoulder..$^{27,33,34}$ One single operator prepared the cavities using a dental surveyor (Paraflex, BEGO GmbH, Bremen, Germany). The teeth were first prepared using $80 \mu \mathrm{m}$ grit diamond burs (837KR.314.012*, 847KR.314.016*, Komet, Dental, Brasseler, Lemgo, Germany) and finished with 30-40 $\mu \mathrm{m}$ grit diamond burs (8837KR.314.012*, 8847 KR.314.016*, 8390.204.016* Komet). ${ }^{35}$

As for milling parameters, $40 \mu \mathrm{m}$ for the spacer and $20 \mu \mathrm{m}$ for the cement gap were established. ${ }^{36}$ Inlay cavities were then scanned using a laboratory scanner (D710 3D Scanner, 3Shape, Copenhagen, Denmark) and CAD software (3D Dental System 2015, 3Shape) was used in order to design the inlays. A CAM unit (Coritec 550i, Imes-icor $\mathrm{GmbH}$, Eiterfeld, Germany) milled the inlays out of the test materials for each tooth.

Adhesive cementation

Inlay restorations were adhesively cemented (Panavia F2.0, Kuraray Noritake Dental Inc., City, Japan) to the corresponding cavities following the manufacturer's instructions. Initially, intaglio surfaces of the inlays were air-abraded (50 micron $\mathrm{Al}_{2} \mathrm{O}_{3}$, Microetcher, Danville Engineering, CA, USA) and then etched with $40 \%$ phosphoric acid (K Etchant gel, Kuraray Noritake Dental Inc.) for $5 \mathrm{~s}$. Equal amounts of silane was mixed (Clearfil SE Bond, Porcelain Bond Activator, Kuraray Noritake Dental Inc.) and applied one coat using a microbrush. The tooth surface was conditioned using a self-etch primer (ED Primer A and B, Kuraray) for $30 \mathrm{~s}$ and air-dried gently with oil-free air. Equal amounts of the base and activator of dualpolymerized resin cement was mixed for $20 \mathrm{~s}$ and the mixture of paste was applied on the intaglio surface of the inlays. The inlay was cemented with the aid of a sticky holder (Vivastick, Ivoclar Vivadent) under 50 $\mathrm{N}$ and excess cement was removed using dental probe and microbrush. The inlay was then photopolymerized from mesial, distal and occlusal aspects for 20 s per surface using an LED unit (Bluephase, 
Ivoclar Vivadent; Light output: $1200 \mathrm{mw} / \mathrm{cm}^{2}$ ). Glycerine gel was applied at the margins (Oxyguard, Kuraray Noritake Dental Inc.), waited for 60 s and rinsed with copious water.

Cement thickness measurement

The cement thickness at the margins and internal surface of the inlays were measured using highresolution 3D micro-CT (SkyScan 1172, Bruker-micro-CT, Kontich, Belgium). The X-ray tube was

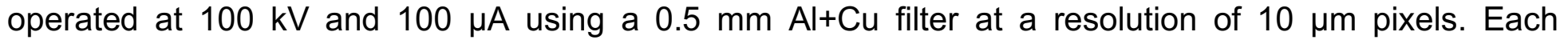
specimen was scanned for a total of 60 minutes at rotation $180^{\circ}$ around the vertical axis. The camera exposure time was $1400 \mathrm{~ms}$, with a rotation step of 0.40 , average frame of 3 and random movement of $20 \mathrm{~mm}$. System reconstruction software (NRecon v.1.6.3, SkyScan, Brucker-micro-CT) was then employed in order to reconstruct the axial cross-sections of the resulting two-dimensional images (8-bit TIFF) with a beam hardening correction of $55 \%$, smoothing of 3 , and an attenuation coefficient range of $0-0.064000$. Thereafter, 3D reconstructions were developed using the softwares to produce the linear and volumetric analysis (CTAn v.1.12 and CT Vol v.2.2.1, Skyscan, Bruker-micro-CT). Finallly, gingival cement thickness was calculated for marginal area and mean axial and pulpal cement thickness were calculated for internal cement thickness (Fig. 1).

Statistical analysis

Statistical analysis was performed with SPSS Statistics for Windows Version 20 (SPSS, Chicago, IL, USA). Kolmogorov-Smirnov and Shapiro-Wilk tests were used to test normal distribution of the data. As the data $(\mu \mathrm{m})$ were normally distributed, one-way analysis of variance (ANOVA) and Tukey`s tests were used. $P$ values less than 0.05 were considered significant in all tests.

\section{Results}

The mean marginal and internal cement thickness were not significant in all inlay materials $(p>0.05)$. 
The marginal cement thickness $(\mu \mathrm{m})$ was the lowest for IPS group $(67.54 \pm 10.16)$ followed by VE $(84.09 \pm 3.94)$ and CS $(95.18 \pm 10.58)$ (Table 2) $(p>0.05)$.

The internal cement thickness $(\mu \mathrm{m})$ was the lowest in CS group $(54.85 \pm 6.94)$, followed by IPS $(60.58 \pm 9.22)$ and $\operatorname{VE}(77.53 \pm 12.13)(p>0.05)$

\section{Discussion}

This study was undertaken in order to compare the compare the marginal and internal cement thickness of molar inlay restorations made of a variety of CAD/CAM materials using an x-ray micro-CT. Based on the results obtained, since there were no significant difference between the measurement areas (marginal versus internal) in all tested materials, the null hypothesis was accepted.

The non-significant differences between groups could also indicate accurate preparation designs. The geometry of the preparation employed in this study was in accordance with the recommended preparation guidelines for ceramic partial coverage restorations..$^{27,33,34}$ In an attempt to standardize the preparation procedures, the same operator working with the same settings and using the dental surveyor prepared the cavities. In addition, the same CAM equipment and software were used to scan the specimens, design the inlays, and mill the restorations.

Clinically acceptable marginal gap of fixed restorations is yet to be formally identified. However, the American Dental Association (ADA) Specification No. 8 states that the thickness of luting cement used to bond a crown should not exceed $40 \mu \mathrm{m}$ when using different type of luting agents. ${ }^{37}$ Although marginal openings in this range are seldom achieved, a $40 \mu \mathrm{m}$ thickness of the luting cement is widely acknowledged as the clinical goal. According to existing research in this area, marginal/internal gap values between 20 and $200 \mu \mathrm{m}$ are found largely clinically acceptable. ${ }^{8,23-28} \mathrm{~A}$ review of studies similar to the current research that have employed an x-ray micro-CT technique revealed marginal/internal gaps between 11.86 and $230.53 \mu \mathrm{m} .{ }^{15-22}$ In the present study, 3D marginal and internal mean cement thicknesses of restorations in all material groups ranged between $60.58 \pm 9.22$ and $95.18 \pm 10.58 \mu \mathrm{m}$, which 
is less than $100 \mu \mathrm{m}$ and, therefore, clinically acceptable. ${ }^{31,32}$ Even the highest cement thickness values in this study were less than those reported in previous studies. This could be attributed to the measurement methodology.

Other methods such as silicon weight and density evaluation, ${ }^{38}$ the use of a triple scan protocol with a non-contact scanner and specific software to perform a virtual 3D analysis, ${ }^{39,40}$ and micro-CT evaluation, ${ }^{41}$ suffer from two major methodological limitations in this domain. The first limitation concerns the number of measurement points where increased number of points on the entire periphery or volume of the restoration can provide a more accurate average assessment of pertinent adaptation. The second methodological limitation relates to the geometric tracking system that defines the limits of the marginal gap measured. ${ }^{42,43}$ 3D assessment using X-ray micro-CT in this study successfully overcomes these limitations. The methodology employed represented a non-destructive approach by which it was possible to perform a quantitative analysis of the marginal and internal accuracy of the restoration in a manner that left the tooth intact and provided means through which the measurements could be reproduced at different time intervals. ${ }^{15-22}$

In this study only one type of inlay preparation was assessed. Other preparation designs for inlays or partial coverage restorations made of CAD/CAM materials may show different results and validity of microCT should also be verified in other preparation designs. It has to be noted that except for IPS, the other CAD/CAM materials used in this study did not require the sintering. Marginal cement thickness in IPS group was less than those with VE and CS, yet being not significant. Whether less cement thickness in marginal area with IPS is a result of sintering or not need to be investigated in future studies. 


\section{Conclusions}

Using 3D micro-CT, marginal and internal cement thickness of MOD inlays manufactured using monolithic lithium disilicate, polymer-infiltrated ceramic and nano-ceramic CAD/CAM materials, presented mean values less than $100 \mu \mathrm{m}$, which could be considered clinically acceptable.

\section{Acklowledgements}

This study was supported by Kırıkkale University, Research Center, Turkey.

\section{Conflict of interest}

The authors did not have any commercial interest in any of the materials used in this study. 


\section{References}

1. Frankenberger $\mathrm{R}$, Petschelt $\mathrm{A}$, Kramer $\mathrm{N}$ : Leucite-reinforced glass ceramic inlays and onlays after six years: clinical behavior. Oper Dent 2000;25:459-465.

2. Pallesen U, van Dijken JW: An 8-year evaluation of sintered ceramic and glass ceramic inlays processed by the Cerec CAD/CAM system. Eur J Oral Sci 2000;108:239-246.

3. El-Mowafy O, Brochu JF: Longevity and clinical performance of IPS-Empress ceramic restorations--a literature review. J Can Dent Assoc. 2002;68:233-237.

4. Hayashi M, Yeung CA: Ceramic inlays for restoring posterior teeth. Aust Dent J 2004;49:60.

5. Fron Chabouis $\mathrm{H}$, Smail Faugeron V, Attal JP: Clinical efficacy of composite versus ceramic inlays and onlays: a systematic review. Dent Mater 2013;29:1209-1218.

6. Zahran M, El-Mowafy O, Tam L, et al: Fracture strength and fatigue resistance of all-ceramic molar crowns manufactured with CAD/CAM technology. J Prosthodont 2008;17:370-377.

7. Kois DE, Isvilanonda V, Chaiyabutr $Y$, et al: Evaluation of fracture resistance and failure risks of posterior partial coverage restorations. J Esthet Restor Dent 2013;25:110-122.

8. Qualtrough AJ, Piddock V, Kypreou V: A comparison of two in vitro methods for assessing the fitting accuracy of composite inlays. Brit Dent J 1993;174:450-454.

9. Frankenberger R, Sindel J, Kramer $\mathrm{N}$, et al: Dentin bond strength and marginal adaptation: direct composite resins vs ceramic inlays. Oper Dent 1999;24:147-155.

10. Addi S, Hedayati-Khams A, Poya A, et al: Interface gap size of manually and CAD/CAM-manufactured ceramic inlays/onlays in vitro. J Dent 2002;30:53-58.

11. Deng $Y$, Miranda P, Pajares A, et al: Fracture of ceramic/ceramic/polymer trilayers for biomechanical applications. J Biomed Mater Res Part A 2003;67:828-833.

12. Coelho PG, Silva NR, Bonfante, EA et al: Fatigue testing of two porcelain-zirconia all-ceramic crown systems. Dent Mater 2009;25:1122-1127. 
13. Zhang $\mathrm{Y}, \mathrm{Kim}$ JW, Bhowmick $\mathrm{S}$, et al: Competition of fracture mechanisms in monolithic dental ceramics: flat model systems. J Biomed Mater Res Part A 2009;88:402-411.

14. Nawafleh NA, Mack F, Evans J, et al: Accuracy and reliability of methods to measure marginal adaptation of crowns and FDPs: a literature review. J Prosthodont 2013;22:419-428.

15. Gonzalo E, Suarez MJ, Serrano B, et al: Comparative analysis of two measurement methods for marginal fit in metal-ceramic and zirconia posterior FPDs. Int J Prosthodont 2009;22:374-347.

16. Pelekanos S, Koumanou M, Koutayas SO, et al: Micro-CT evaluation of the marginal fit of different InCeram alumina copings. Eur J Esthet Dent 2009;4:278-292.

17. Borba M, Cesar PF, Griggs JA, et al: Adaptation of all-ceramic fixed partial dentures. Dent Mater 2011;27:1119-1126.

18. Schladitz K: Quantitative micro-CT. J Microscopy 2011;243:111-117.

19. Raigrodski AJ, Hillstead MB, Meng GK, et al: Survival and complications of zirconia-based fixed dental prostheses: a systematic review. J Prosthet Dent 2012;107:170-177.

20. Neves FD, Prado CJ, Prudente MS, et al: Micro-computed tomography evaluation of marginal fit of lithium disilicate crowns fabricated by using chairside CAD/CAM systems or the heat-pressing technique. J Prosthet Dent 2014;112:1134-1140.

21. Alfaro DP, Ruse ND, Carvalho RM, et al: Assessment of the internal fit of lithium disilicate crowns using micro-ct. J Prosthodont 2015;24:381-386.

22. Pimenta MA, Frasca LC, Lopes R, et al: Evaluation of marginal and internal fit of ceramic and metallic crown copings using x-ray microtomography (micro-CT) technology. J Prosthet Dent 2015;114:223-228.

23. Denissen H, Dozic A, van der Zel J, et al: Marginal fit and short-term clinical performance of porcelainveneered CICERO, CEREC, and Procera onlays. J Prosthet Dent 2000;84:506-513.

24. Krifka $S$, Anthofer $T$, Fritzsch $M$, et al: Ceramic inlays and partial ceramic crowns: influence of remaining cusp wall thickness on the marginal integrity and enamel crack formation in vitro. Oper Dent 2009;34:32-42. 
25. McLean JW, von Fraunhofer JA: The estimation of cement film thickness by an in vivo technique. Brit Dent J 1971;131:107-111.

26. Marxkors R: [Marginal seal of cast crowns]. Dtsch Zahnarztl Z.1980;35:913-915.

27. Stappert CF, Chitmongkolsuk S, Silva NR, et al: Effect of mouth-motion fatigue and thermal cycling on the marginal accuracy of partial coverage restorations made of various dental materials. Dent Mater $2008 ; 24: 1248-1257$.

28. Vanlioglu BA, Evren B, Yildiz C, et al: Internal and marginal adaptation of pressable and computeraided design/computer-assisted manufacture onlay restorations. Int J Prosthodont 2012;25:262-264.

29. Torrado E, Ercoli C, Al Mardini M, et al: A comparison of the porcelain fracture resistance of screwretained and cement-retained implant-supported metal-ceramic crowns. J Prosthet Dent 2004;91:532-537.

30. http://www.gcamerica.com/products/operatory/CERASMART/index.php. 2015

31. Kramer N, Lohbauer U, Frankenberger R: Adhesive luting of indirect restorations. Am J Dent 2000;13:60D-76D.

32. Federlin M, Krifka S, Herpich M, et al: Partial ceramic crowns: influence of ceramic thickness, preparation design and luting material on fracture resistance and marginal integrity in vitro. Oper Dent $2007 ; 32: 251-260$.

33. Karakaya S, Sengun A, Ozer F: Evaluation of internal adaptation in ceramic and composite resin inlays by silicon replica technique. J Oral Rehabil 2005;32:448-453.

34. Ahlers MO, Morig G, Blunck U, et al:. Guidelines for the preparation of CAD/CAM ceramic inlays and partial crowns. Int J Comp Dent 2009;12:309-325.

35. Stappert CF, Denner N, Gerds T, et al: Marginal adaptation of different types of all-ceramic partial coverage restorations after exposure to an artificial mouth. Brit Dent J 2005;199:779-783..

36. Reich S, Gozdowski S, Trentzsch L, et al: Marginal fit of heat-pressed vs. CAD/CAM processed allceramic onlays using a milling unit prototype. Oper Dent 2008;33:644-650. 
37. American Dental Association: ANSI/ADA Specification No. 8 for zinc phosphate cement. In: Guide to Dental Materials and Devices (ed 5), 1970-1971.

38. Alghazzawi TF, Liu PR, Essig ME: The effect of different fabrication steps on the marginal adaptation of two types of glass-infiltrated ceramic crown copings fabricated by CAD/CAM technology. J Prosthodont 2012;21:167-172.

39. Matta RE, Schmitt J, Wichmann M, et al: Circumferential fit assessment of CAD/CAM single crowns--a pilot investigation on a new virtual analytical protocol. Quintessence Int 2012;43:801-809.

40. Schaefer O, Watts DC, Sigusch BW, et al: Marginal and internal fit of pressed lithium disilicate partial crowns in vitro: a three-dimensional analysis of accuracy and reproducibility. Dent Mater 2012;28:320-326. 41. Boitelle $P$, Mawussi B, Tapie $L$, et al: A systematic review of CAD/CAM fit restoration evaluations. J Oral Rehabil 2014;41:853-874.

42. Persson A, Andersson M, Oden A, et al: A three-dimensional evaluation of a laser scanner and a touch-probe scanner. J Prosthet Dent 2006;95:194-200.

43. Colpani JT, Borba M, Della Bona A: Evaluation of marginal and internal fit of ceramic crown copings. Dent Mater 2013;29:174-180. 


\section{Captions to tables and legends:}

Tables:

Table 1. Brands, abbreviations, manufacturers and batch numbers of the inlay materials used in this study.

Table 2. Mean marginal and internal cement thickness and standard deviations (SD) ( $\mu m$ ) for the MOD inlay restorations made of different CAD/CAM materials. For group abbreviations see Table 1.

Figures:

Figure 1 Micro-CT images of cement thickness under the inlays in MOD cavities from axial, occlusal and mesial views. 
Tables:

\begin{tabular}{ccc}
\hline Brand & Manufacturer & Batch Numbers \\
\hline IPS e-max CAD & Ivoclar Vivadent GmbH, \\
(IPS) & Schaan, Liechtenstein \\
Vita Enamic & \\
(VE) & VITA Zahnfabrik, GmbH \& Co, \\
Cerasmart & Bad Säckingen, Germany & 41960 \\
(CS) & GC Dental Products, \\
& Tokyo, Japan & 1412081 \\
\hline
\end{tabular}

Table 1. Brands, abbreviations, manufacturers and batch numbers of the inlay materials used in this study.

\begin{tabular}{ccc}
\hline Experimental Groups & $\begin{array}{c}\text { Marginal Cement thickness } \\
( \pm \mathbf{S D})(\boldsymbol{\mu m})\end{array}$ & $\begin{array}{c}\text { Internal Cement thickness } \\
( \pm \mathbf{S D})(\boldsymbol{\mu m})\end{array}$ \\
\hline IPS & $67.54 \pm 10.16$ & $60.58 \pm 9.22$ \\
VE & $84.09 \pm 3.94$ & $77.53 \pm 12.13$ \\
CS & $95.18 \pm 10.58$ & $54.85 \pm 6.94$ \\
& $p>0.05$ & $p>0.05$ \\
\hline
\end{tabular}

Table 2. Mean marginal and internal cement thickness and standard deviations (SD) ( $\mu \mathrm{m})$ for the MOD inlay restorations made of different CAD/CAM materials. For group abbreviations see Table 1. 


\section{Figures:}

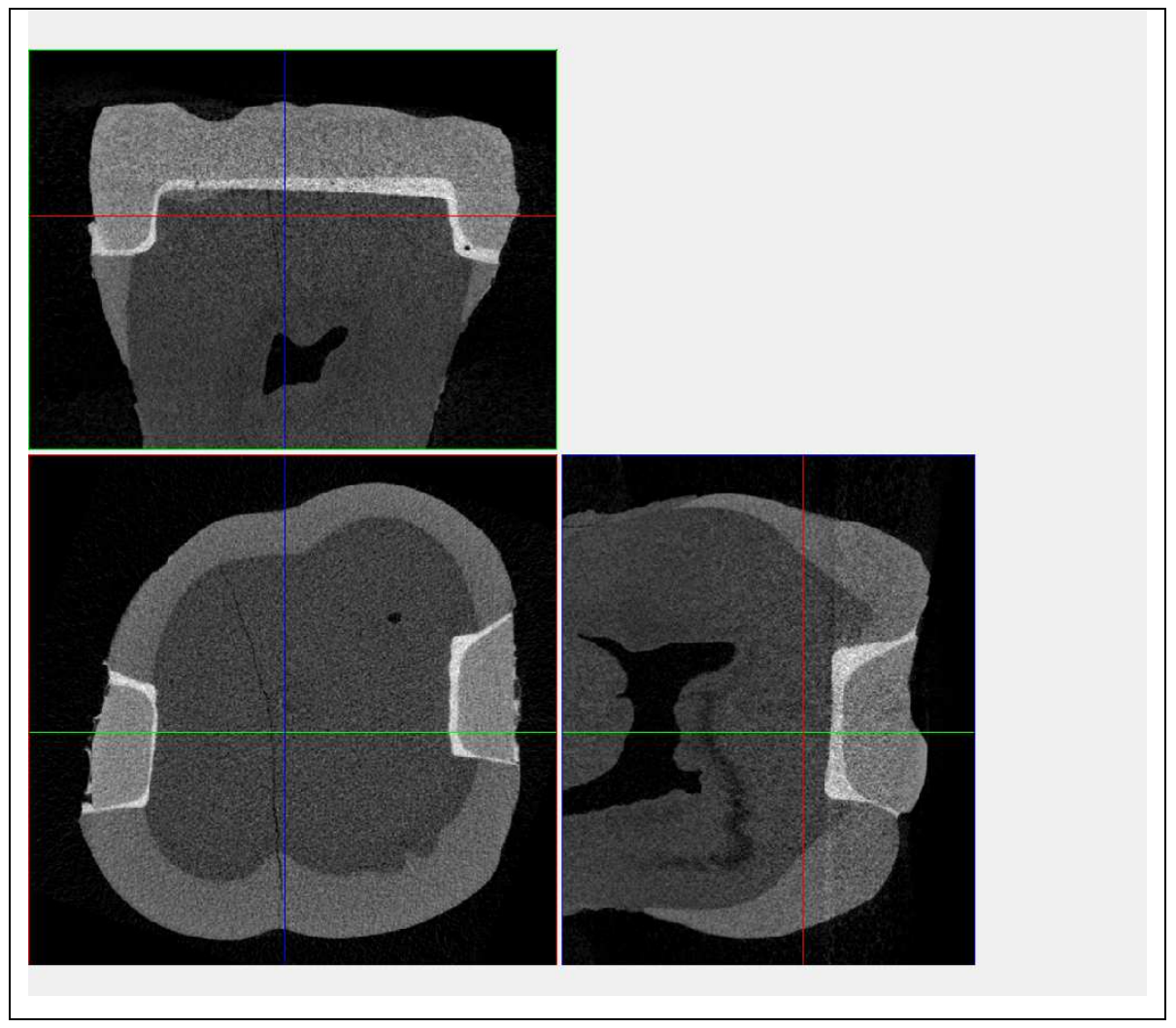

Figure 1 Micro-CT images of cement thickness under the inlays in MOD cavities from axial, occlusal and mesial views. 